\title{
Barrier Analysis Analysed in MORT Perspective
}

\author{
John Kingston, Robert Nertney, Rudolf Frei and Philippe Schallier \\ Noordwijk Risk Initiative Foundation \\ Delft, Netherlands \\ Floor Koornneef \\ Safety Science Group, Delft University of Technology \\ Delft, Netherlands
}

\begin{abstract}
This paper discusses the incorporation into MORT of the Haddon energy-flow notion. It focuses on the relationships between MORT barrier analysis, Energy Trace and Barrier Analysis (ETBA), Control Change Cause Analysis (3CA) and the cybernetic conventions developed by Ashby. The implications for the next generation of MORT and the application of barrier analysis in domains outside of safety are discussed.
\end{abstract}

\section{The barrier concept is intrinsic to MORT}

The barrier concept is intrinsic to the Management Oversight and Risk Tree, in both MORT's manifestation as a description of a safety management programme (e.g. Johnson, 1973) and as a suite of analytical methods, of which the most visible is the MORT logic tree (Frei et al, 2002(a) and 2002(b)). For the most part, MORT analysis is concerned with the energy flows that get things done in a work/process system. A characteristic of MORT analysis is that it operates in the perspective of what people wished to achieve through the work process rather than being focussed solely on what could go wrong.

Within MORT, barrier analysis refers the use of the MORT logic tree to analyse the interaction between a particular energy flow and a vulnerable target (meaning somebody or something), this event being referred to generally as an "energy transfer" (Trost and Nertney, 1995). There may be many such events within the one accident and Energy Trace and Barrier Analysis (ETBA) is used to identify them. 


\section{MORT's origins: the epidemiological model of barriers and the Haddon concept}

The development of MORT (1969-1973) by Johnson and his team was a process of incorporating ideas that could prove helpful in the management of safety at nuclear facilities. Quite often the ideas for MORT came from outside of the nuclear industry and the field of safety. One of the present authors (Nertney) was a member of Johnson's team and recalls that the barrier concept was initially identified in epidemiology, where disease vectors occupy the same role as Haddon's energy flow. The MORT developers adopted Haddon to make barrier analysis recognisable to and usable by the engineers who were their primary audience. This worked but had a downside too. The developers (Johnson and Nertney) could easily see energy as the "basic currency that applies to everything", but sometimes others had problems with this. In this way, the expedient adoption of the Haddon "energy barrier notion" became a minor obstacle itself. As Nertney explains it "Johnson and I had to reexplain the relevance of the idea to settings in which people wouldn't normally think in terms of energy transfers. That meant that we had to do quite a lot of tap-dancing around to show how the energy transfer idea could handle issues like starvation. But it's a problem - people think about food, not about cellular metabolism".

\section{The role of ETBA in investigation}

Most incidents contain more than one event that requires explanation, often there are several. Elsewhere (Frei et al, 2003) we have characterised ETBA as a way of identifying the "norms, novelties and deviations - NNDs" that are of relevance to a particular incident or accident. As we say there:

"When an incident occurs, it sometimes signals failures in the control of an activity or of protective systems. To provide the focus for subsequent [root] causal analysis, these failures can be characterised as deviations from norms. In many cases, identifying norms equates to identifying the standards that applied to a specific instance of control or protection. (Frei et al, 2003).

In the context of MORT root cause analysis, ETBA is used to decompose a sequence of events into discrete events or units, each of which can be the subject of the analysis. In effect, each row of an ETBA is a unit of MORT analysis and this makes the decomposition through ETBA a prerequisite for the investigative use of MORT. This prerequisite can be argued to apply to root cause methods generally. Without clear delineation of events, it is difficult for the analyst to settle on what it is that they are trying to explain through root cause analysis.

Within the one accident, the "units of analysis" may vary widely in the NNDs that are revealed for root cause analysis. Furthermore, each NND can reveal different patterns of causation; a root cause analysis specific to each NND allows a finely grained picture (and hopefully well-evidenced) to be established. Often MORT 
analysis of several rows from an $\mathrm{ETBA}^{1}$ will reveal recurrent general themes (i.e. converging root causes). However, although the general themes may be interesting in themselves, the specific grounds from which they arise provide a firmer basis on which to make recommendations. Root cause analysis that is not focussed in this way can only produce very impressionistic causal themes but without a way of connecting the general to the specific; a recipe for management hand-wringing but not for definite, targeted remedial action.

ETBA can also be applied on its own account in investigations as a standalone method. Used this way, ETBA can produce a very concise summary of the significant events and the barriers and controls implicated in the accident.

\section{Reconciling qualities of flexibility and reproducibility in Barrier Analysis}

ETBA is a powerful idea, and a flexible tool, but as Johnson (1973) points out, a key to this potential is rigour of application. The rigorous use of ETBA has three aspects:

(1) the "meticulous trace" aspect requires the analyst to identify all the energy transfers occurring in the sequence of events (meaning within the accident, incident, and precursor events). Each unwanted energy transfer begs the question "where did this energy come from?" a question that Johnson tells us must be repeatedly asked and answered;

(2) barriers and controls need to be identified precisely and in context. Generic statements about barriers and controls cannot be analysed properly in terms of the standards, or the test of reasonableness, that apply to the context of the accident;

(3) the same level of system needs to be maintained when associating an energy transfer with the barriers and controls that correspond to it. For example an electric shock suffered by an Electrician from a live system, could correspond to barriers and controls such as "isolate from electrical supply". Sometimes, novice analysts "jump" immediately to higher order controls such as training or risk assessment for zeroorder energy transfers. Training will not stop the flow of current, but training may have a role in implementing the "isolation".

The points above are some of the grounds for a rigorous approach, but also worthy of note is the desire by the developers of MORT to combine rigorous analysis with a flexible approach, that is, one not limited to a literal view of energy flows. Their experience was that overly-rigid analyses can block valuable insights into problems. For example, if one adopts a form of barrier analysis that is not restricted to energy

\footnotetext{
${ }^{1}$ The practical relationship between ETBA and MORT analysis of barriers is fully explained in the MORT Users Manual (Frei et al, 2002).
} 
transfers, one can also think in terms of "how did bad things get into our system" and "why good things did not get into our system". For example, having purchase orders signed-off by safety professionals provides a way of blocking "bad things" from entering your system - looking at a purchase order will often set-off alarm bells in the safety professional's mind. One might even imagine extending barrier analytical thinking to the recommendation phase of accident investigation: to consider what barriers might exist to implementing recommendations.

If we are to apply ETBA thinking to forms of barrier analysis that use analogues of the energy transfer concept, rigorous analysis will require appropriate translation of the three aspects mentioned earlier: meticulous trace, specific to context and operate at one-level of system.

\section{Two forms of barrier analysis: ETBA and 3CA}

Our experience of designing a new form of barrier analysis (to serve as the basis of the 3CA method, Kingston, 2003) provides further insight into the general principles. We wanted the new form of barrier analysis to have a good degree of reproducibility and to enable analysts to capture issues without the need for "forcing" the analysis.

In the event, we used "agent of change" instead of "energy flow". We arrived at "agent of change" by using the cybernetics model devised by Ashby (1956). Ashby's scheme proposes four functions, related as follows: a disturbance (D) acts upon a transformational system $(\mathrm{T})$ in a way that would adversely change the values of one or more essential variables (E) were it not for the action of a regulator $(\mathrm{R})$. It needs to be recognised that these functions are not necessarily separate entities, and indeed will quite often be found in the one entity.

Although "disturbance" is defined by the adverse effect on essential variables, it is best seen in a wider context. What Ashby calls "disturbance" (D) is only one functional aspect of the entire input to the transformational system (T) most of which is beneficial to maintaining the essential variables (E) within their desired range of values. The analytical perspective is of a system achieving something useful rather than a just as a convention for stating problems. This is highly congruent with the MORT viewpoint discussed earlier in section one. In terms of the Ashby model, this perspective reminds us of the need to ensure that $\mathrm{R}$ (by acting on $\mathrm{T}$ ) does not unduly obstruct the generally beneficial flow of input of which D is a problematic subset. Seen this way, a desirable property in a regulator is that it should be highly selective about what it blocks. An "undiscerning" regulator, one that blocks too much "beneficial" input, might itself have an adverse impact on the essential variables.

Returning to the relationship between the Ashby model and 3CA, the headings we used in the new barrier analysis are: (1) Change to person or thing; (2) Agent of 
change; (3) Adverse effect of change; (4) Work controls or protective barriers implicated in (1)/(2). The correspondence between these headings and Ashby's conventions is illustrated below in Figure 1.

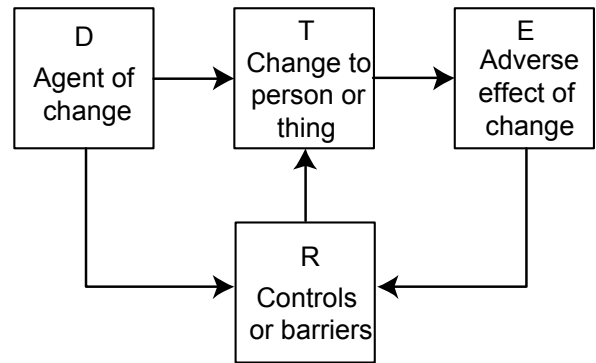

Figure 1: Correspondence of 3CA Headings and Ashby conventions (configured in a full-information paradigm)

In 3CA, instead of the "meticulous energy trace" of ETBA, the barrier analysis is performed on a robust, sequenced description of what happened and how (as could be obtained by using a method like ECFA+ (Kingston et al. 2004) or STEP (Kendrick and Benner, 1987). With the sequenced description available, the 3CA process begins by identifying all events that create an adverse change in control or that increase risk - these terms often being the two sides of the same coin. Kingston (2002) provides a comparison of the barrier analytical terms used in ETBA and $3 \mathrm{CA}$.

\section{The next horizon of generalisation for MORT-based barrier analysis}

Haddon's energy transfer model is a special instance of a more general concept. A great advantage of Haddon's model is the facility for rigorous application with the clarity of insight and confidence that this can bring to barrier analysis. However, these benefits are available at some cost to flexibility with what Energy Trace and Barrier Analysis can be applied to. Arguably anything can be stated in terms of energy flow, but this can sometimes lead to very forced analyses and tenuous arguments.

When Johnson and Nertney saw how an idea from epidemiology could apply in the context of the nuclear industry, and adopted Haddon's scheme "to make it stick", they were using an analogy between those two worlds. We believe that the Ashby model, and the ideas to which it connects, provide the underlying set of concepts to these and other worlds of application. This has two outcomes: first, the ability to develop barrier analyses tailored to any domain of risk management, and second; the ability to make formal comparisons between barriers and controls in one domain and another, even if they are superficially very dissimilar. 
One immediate conclusion of this line of thinking is that barrier analysis is essentially about understanding the selective blocking of information in a specific context, whether the analyst is concerned with physical blocking of an energy flow by a barrier, or blocking of terrorist actions using intelligence generated by a security agency. Both MORT and the Ashby models remind us of the need for selectivity in these arrangements: block the harm but not the work, obstruct the terrorist but not the citizen.

We will be applying these insights in the next generation of MORT, making it usable in domains of risk management beyond health, safety and environmental protection.

\section{References}

Ashby, W.R. (1956). Introduction to Cybernetics. London, Chapman and Hall.

Frei, R., Kingston, J., Koornneef, F., and Schallier, P. (2002a), NRI MORT User's Manual. Ref. NRI-1 (2002), Pub. Noordwijk Risk Initiative Foundation, The Netherlands. www.nri.eu.com.

Frei, R., Kingston, J., Koornneef, F., and Schallier, P. (2002b), NRI MORT Chart. Ref. NRI2 (2002), Pub. Noordwijk Risk Initiative Foundation, The Netherlands. www.nri.eu.com.

Frei, R., Kingston, J., Koornneef, F., and Schallier, P. (2003) Investigation Tools in Context. JRC/ESReDA Seminar on Safety Investigation of Accidents on 12-13 May 2003, Petten, The Netherlands.

Hendrick, K. and Benner, L. (1987), Investigating accidents with STEP. Marcel Dekker.

Johnson, W.G. (1973). MORT - The Management Oversight and Risk Tree. SAN 821-2. US Atomic Energy Commission.

Kingston, J. (2002) 3CA - Control Change Cause Analysis Manual. NRI-3 (2002), Pub. Noordwijk Risk Initiative Foundation, The Netherlands. www.nri.eu.com.

Kingston, J., Jager, J., and Koornneef, F. (2004) ECFA+: Events and Conditional Factors Analysis. Ref. NRI-4 (2004), Pub. Noordwijk Risk Initiative Foundation, The Netherlands. www.nri.eu.com.

Trost, W.A. and Nertney, R.J. (1995), Barrier Analysis. US Department of Energy Ref. DOE 76-45/29, SSDC-29. 